Journal of Computer Science 8 (2): 181-187, 2012

ISSN 1549-3636

(C) 2012 Science Publications

\title{
Adhoc on Demand Multipath Reliable and Energy Aware Quality of Service Routing for Mobile Adhoc Networks
}

\author{
${ }^{1}$ Thilagavathe, V. and ${ }^{2} \mathrm{~K}$. Duraiswamy \\ ${ }^{1}$ Department of Computer Science, \\ Institute of Road and Transport Technology, Erode, Tamilnadu, India \\ ${ }^{2}$ Department of Computer Science and Engineering, \\ K.S. Rangasamy College of Technology, Tiruchengode, Tamilnadu, India
}

\begin{abstract}
Problem statement: In a mobile adhoc network, there are a number of challenges for providing quality of service routing for multimedia applications and real time applications like voice transmission. The QoS parameters considered in this study are link reliability and energy consumption. Existing studies estimate the link reliability constraint between two nodes using exponentially distributed epoch lengths. The major problem in this approach is that the nodes are having memoryless property and the hazard rate is independent of time. Approach: In study, an analytical expression is proposed to estimate the link reliability of MANET when the nodes have identical weibull distribution where the hazard rate is a function of the time. Adhoc On Demand Multipath Reliable and Energy Aware QoS Routing (AOMP-REQR) protocol is designed by including the QoS constraints (Link Reliability and Energy Consumption) in the existing protocol AOMDV, which finds the multiple link disjoint stable paths. Results: Simulation results show that the proposed algorithm achieves better packet delivery ratio with reduced energy consumption and delay. Conclusion: Since the proposed analytical method estimates the link reliability in better way, AOMP-REQR protocol finds the multiple link disjoint stable paths and also the energy consumption is less.
\end{abstract}

Key words: Link reliability, energy consumption, weibull, quality of service, stable paths, analytical expression, mobile adhoc network, epoch lengths, hazard rate, weibull distribution

\section{INTRODUCTION}

Traditional wireless and cellular offer a limited range of mobile communication and are constrained in their deployment by their need for underlying network infrastructure. Demands for user mobility have created interest in the development of an emerging class of self organizing, rapidly deployable network architecture called Mobile Adhoc Networks. Mobile Adhoc Networks consist of several mobile nodes that are capable of communicating with each other without the use of a network infrastructure. Since MANETs are infrastructure less, each node acts as a router forwarding data packets to other nodes and it can be used for emergency situations like military applications, disaster relief and emergency services.

In MANETs routes between nodes may include multiple hops and hence are called multi hop networks. Each mobile node can communicate with other mobile node in its communication range, if the node is beyond its range the node needs other intermediate nodes to relay information (i.e., each node act as a router to forward information to other nodes). MANET routing becomes difficult, when nodal mobility causes the breakdown of radio links, how to select reliable paths that can last as long as possible becomes an issue for routing and another is how to select energy constrained path.

In this study, an analytical method is proposed to estimate the link reliability when the nodes have identical weibull distribution where the hazard rate is a function of the time. With the inclusion of multiple constraints link reliability and energy consumption in AOMDV several link disjoint stable paths are found from a source to destination.

Related work: The Ad hoc On Demand Distance Vector AODV routing protocol provides on demand route discovery in mobile ad hoc networks (Perkins and

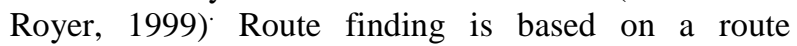
discovery cycle involving a broadcast network search and a unicast reply containing discovered paths. AODV relies on per node sequence number for loop freedom and for ensuring selection of most recent routing path. Ad hoc

Corresponding Author: Thilagavathe, V., Department of Computer Science, Institute of Road and Transport Technology,

Erode, Tamilnadu, India 
On Demand Multipath Distance Vector (AOMDV) routing protocol is an extension of AODV routing protocol. It is used to compute multiple paths during route discovery. It is designed primarily for dynamic ad hoc networks. The AOMDV protocol applies a route update rule to establish and maintain multiple loop free routes at each node (Marina and Das, 2001). A distributed protocol is used to find link disjoint paths.

A probability link model which can predict the future status of a wireless link has been discussed in $\mathrm{Su}$ and Gerla, (1999) In Lim et al., (2002); Meghanathan, (2007) authors make use of statistical prediction based on the node movement. In this approach, a link stability probability has been defined on the basis of the random mobility model. A formal model to predict the life time of a routing path based on random walk mobility and on the prediction technique was proposed in Maleki et al. (2003). It considers the probability model derived through the subdivision into cells area where mobile nodes move and on the observations of node movements in these cells. Transition probabilities are calculated and a state based model of the movement among the cells is considered. Each connection between a mobile node in cell and the other mobile nodes among its neighbor cells is considered as the state of the wireless link. In this way, the wireless link dynamic is determined between a mobile node and its neighbors, permitting the calculation of the link lifetime. After this, through the assumption of independent link failure, the route breakage probability is derived. In Jiang et al. (2001), a prediction based estimation algorithm tries to predict the probability that an active link between two nodes will be continuously available for a certain period $\mathrm{T}_{\mathrm{P}}$, which is obtained based on the current node's movement. The only assumption used for the $T_{P}$ prediction is that both nodes associated with the link will keep their current velocities. In Jiang, (2004), calculates $L\left(T_{P}\right)$ by considering all possible changes in velocities occurring during period $\mathrm{T}_{\mathrm{P}}$. The method was proposed by (Balaji and Duraisamy, 2011), identifies how the network topology changes based on a node and its next one hop nodes. Computation of power of each node within one hop distance of the destination node is computed periodically and used to identify whether nodes are converging or diverging. Based on the convergence or divergence of nodes an algorithm is proposed to modify the Active Route Timeout.

The MTPR (Minimum Total Transmission

Power Routing) (Scott and Bambos, 1996) was proposed to minimize the total transmission power consumption of nodes participating in the acquired route. Since the MTPR does not consider the remaining power of nodes, it fails to prolong the lifetime of each host. Min-Max Battery Cost Routing (MM-BCR) (Singh et al., 1998) considers the remaining power of nodes as the metrics for acquiring routes in order to prolong the life time of each node. (Toh, 2001) proposed the Conditional Max-Min Battery Capacity Routing (CMMBCR) protocol, is a hybrid protocol that tries to arbitrate between the MTPR and MMBCR. (Maruthamuthu and Sankaralingam, 2011), proposed the new protocol EMQRPDM with multiple QoS constraints between source and destination. It mainly considers power constraint of nodes for efficient packet transmission and also a new mobility prediction formula is used for PredictedLET calculation to select a stable path with minimal cost. It provides a quick response to changes in the network reduces the waste of network resources and produces significant improvement in data transmission rate and hence reduces control overhead for reconstructing a routing path. In (Thilagavathe and Duraiswamy, 2010), Reliable and Energy ware QoS Routing protocol for Mobile Adhoc Networks (REQR) was proposed to find the maximum link reliable and minimally energy consumed multiple paths between the source and the destination.

\section{MATERIALS AND METHODS}

Estimation of link reliability: A major parameter in link reliability estimation is the epoch length of nodes. An epoch is a random length interval during which a node moves in constant direction at a constant speed. A usual assumption made is that the epoch lengths are identically independent exponentially (memory less) distributed random variable, thus having a constant hazard rate; though link reliability is time dependent phenomena and it is far from reality. Thus in this study prediction based reliability estimated in MANETs weibull nodes.

Given a prediction $t_{p}$ on the continuously available time for an active link between two nodes at time $t_{0}$, the conditional reliability $\mathrm{CR}\left(\mathrm{t}_{\mathrm{p}}\right)$ is calculated as:

$$
\mathrm{CR}\left(\mathrm{t}_{\mathrm{p}}\right)=\mathrm{CR}_{0}\left(\mathrm{t}_{\mathrm{p}}\right)+\mathrm{CR}_{\mathrm{k}}\left(\mathrm{t}_{\mathrm{p}}\right)
$$

$\mathrm{CR}_{0}\left(\mathrm{t}_{\mathrm{p}}\right)=$ Link reliability when velocities of the two nodes are not varied during the interval $\left(\mathrm{t}_{0}, \mathrm{t}_{0+} \mathrm{t}_{\mathrm{p}}\right)$

$\mathrm{CR}_{\mathrm{k}}\left(\mathrm{t}_{\mathrm{p}}\right)=$ Link reliability when $\mathrm{k}$ changes in velocities occur during $\left(\mathrm{t}_{0}, \mathrm{t}_{0+} \mathrm{t}_{\mathrm{p}}\right)$

$\mathrm{CR}_{0}\left(\mathrm{t}_{\mathrm{p}}\right)=$ Probability that the epochs from $\mathrm{t}_{0}$ onwards for the two nodes, are longer than $t_{p}$ since $t_{p}$ is an accurate prediction, if the movements of the nodes are unchanged. For each weibull node we have: 


$$
\mathrm{P}\left(\mathrm{T}>\left(\mathrm{t}_{0}+\mathrm{t}_{\mathrm{p}}\right) / \mathrm{T}>\mathrm{t}_{0}\right)=\frac{\mathrm{P}\left(\mathrm{T}>\left(\mathrm{t}_{0}+\mathrm{t}_{\mathrm{p}}\right)\right)}{\mathrm{P}\left(\mathrm{T}>\mathrm{t}_{0}\right)}=\mathrm{e}^{\left.-\alpha\left[\mathrm{t}_{0}+\mathrm{t}_{\mathrm{p}}\right)^{\beta}-\mathrm{t}_{0}{ }^{\beta}\right]}
$$

Since the nodes are independent:

$$
\mathrm{CR}_{0}\left(\mathrm{t}_{\mathrm{p}}\right)=\mathrm{e}^{-2 \alpha\left[\left(\mathrm{t}_{0}+\mathrm{t}_{\mathrm{p}}\right)^{\left.\beta_{-\mathrm{t}_{0}} \beta\right]}\right.}
$$

$\mathrm{CR}_{\mathrm{k}}\left(\mathrm{t}_{\mathrm{p}}\right)$ is calculated as in Jiang (2004), when $\mathrm{k}>0$ indicates that the number of changes in velocities happening during $t_{p}$. The number of velocity changes in a span $t_{p}$ of node is a random process satisfying Poisson postulates with the mean rate of occurrence equal to the reciprocal of the mean epoch. The probability for the link joining nodes $\mathrm{n}_{1}, \mathrm{n}_{2}$ to have $\mathrm{k}$ link epochs in span $\mathrm{t}_{\mathrm{p} \text { : }}$

$$
\mathrm{p}_{\mathrm{k}}\left(\mathrm{t}_{\mathrm{p}}\right)=\mathrm{e}^{-\lambda \mathrm{t}_{\mathrm{p}}} \frac{\left(\lambda \mathrm{t}_{\mathrm{p}}\right)^{\mathrm{k}}}{\mathrm{k}}
$$

where, $\lambda=\lambda_{1}+\lambda_{2}$ a link epoch is the time period during which no change occurs in the velocities of the 2 nodes and is the inter arrival time between two velocity changes. If arrivals are Poisson with mean $\lambda$, then inter arrivals are exponential with mean $\lambda^{-1}$.

Let $\gamma$ be the link epoch random variable. Its density is $\lambda \mathrm{e}^{-\lambda \tau}, \tau>0$ and cdf is $1-\mathrm{e}^{-\lambda \tau}$. These link epochs divide the span $t_{p}$ in $\left(t_{0}, t_{0+} t_{p}\right)$ to $(k+1)$ parts, if $k$ events occur. Let $\mathrm{w}_{\mathrm{i}}$ be the probability that the link is reliable during the $\mathrm{i}^{\text {th }}$ link poch. Then the link reliability for $\mathrm{k}(>0)$ events of independent velocity changes is $\prod_{i=1}^{k} w_{i}$.

The probabilities $\mathrm{w}_{\mathrm{i}}$ depend on the velocities of the nodes during $\tau_{\mathrm{i}}$. Let $\tau_{\mathrm{i}}^{*}$ be the maximum period of $\tau_{\mathrm{i}}$ during hich the link is reliable. This depends on, the maximum allowable distance between the nodes for minimal requirements to maintain communication quality. Hence like $t_{p}, \tau_{\mathrm{i}}^{*}$ should be such that, $\mathrm{d}\left(\mathrm{n}_{1}, \mathrm{n}_{2}\right) \leq \delta$. Given $\delta$ and the velocities during the ith epoch, $\tau_{\mathrm{i}}^{*}$ can be calculated as in Lim et al. (2002).

Clearly, if $\gamma<\tau_{i}^{*}$, then the link is reliable with probability $\left(1-\mathrm{e}^{-\lambda \tau_{\mathrm{i}}^{*}}\right)$ and hence:

$$
\mathrm{CR}_{\mathrm{k}}\left(\mathrm{t}_{\mathrm{p}}\right)=\prod_{\mathrm{i}=1}^{\mathrm{k}}\left(1-\mathrm{e}^{-\lambda \tau_{\mathrm{i}}^{*}}\right)
$$

Thus:

$$
\begin{aligned}
& \operatorname{CR}_{\mathrm{k}}\left(\mathrm{t}_{\mathrm{p}}\right) \mathrm{CR}_{0}\left(\mathrm{t}_{\mathrm{p}}\right)+\sum_{\mathrm{k}=1}^{\infty} \mathrm{CR}_{\mathrm{k}}\left(\mathrm{t}_{\mathrm{p}}\right) \mathrm{P}_{\mathrm{k}}\left(\mathrm{t}_{\mathrm{p}}\right) \\
& =\mathrm{e}-2 \alpha\left[\left(\mathrm{t}_{0}+\mathrm{t}_{\mathrm{p}}\right)^{\beta}-\left(\mathrm{t}_{\mathrm{o}}\right)^{\beta}\right]+\sum_{\mathrm{k}=1}^{\infty} \\
& {\left[\prod_{\mathrm{i}=1}^{\mathrm{K}}\left(1-\mathrm{e}^{-\lambda \tau_{\mathrm{i}}^{*}}\right)\right]\left[\mathrm{e}-\lambda \tau_{\mathrm{p}} \frac{\left(\lambda \tau_{\mathrm{p}}\right)^{\mathrm{k}}}{\mathrm{k}}\right]}
\end{aligned}
$$

This gives the reliability for the link for a given prediction $t_{p}$ at time $t_{0}$ in MANETs with weibull nodes. Calculation of energy consumption: In MANET energy related protocols, usual assumption made on transmission energy is modeled using the Shadowing model (Tan et al., 2007). In this model transmission energy per second can be given by:

$$
\mathrm{p}_{\mathrm{t}}=\gamma \times \mathrm{d}^{\mathrm{r}}
$$

where, $\gamma$ is determined by the frequency of radio, receiver's threshold and signal to noise threshold, $d$ is transmission range and $\mathrm{r}$ is the path loss component. Transmitting RTS and CTS consumes the following total energy. Transmitting RTS and CTS consumes the following total energy:

$$
\begin{aligned}
& \mathrm{E}_{\mathrm{RTS}}=\gamma \times \mathrm{d}_{\text {max }}^{\mathrm{r}} \times \mathrm{T}_{\mathrm{RTS}} \\
& \mathrm{E}_{\mathrm{CTS}}=\gamma \times \mathrm{d}_{\text {max }}^{\mathrm{r}} \times \mathrm{T}_{\mathrm{CTS}}
\end{aligned}
$$

Data and ACK are transmitted using the following energy:

$$
\begin{aligned}
& \mathrm{E}_{\mathrm{DATA}}^{\mathrm{ij}}=\gamma \times \mathrm{d}_{\mathrm{ij}}^{\mathrm{r}} \times \mathrm{T}_{\mathrm{DATA}} \\
& \mathrm{E}_{\mathrm{ACK}}^{\mathrm{ij}}=\gamma \times \mathrm{d}_{\mathrm{ij}}^{\mathrm{r}} \times \mathrm{T}_{\mathrm{ACK}}
\end{aligned}
$$

where, $d_{\max }$ is the maximum transmission range, $d_{i j}$ is the distance between node $\mathrm{i}$ and $\mathrm{j}, \mathrm{T}_{\mathrm{RTS}}, \mathrm{T}_{\mathrm{CTS}}, \mathrm{T}_{\mathrm{DATA}}$, $\mathrm{T}_{\mathrm{ACK}}$ are the transmission time of RTS, CTS, DATA and ACK packets.

$$
\begin{aligned}
& \mathrm{E}_{\mathrm{SD}}=\sum_{\mathrm{K}=1}^{\mathrm{m}-1} \gamma \times \mathrm{d}_{\mathrm{k}}(\mathrm{k}+1) \times\left(\mathrm{T}_{\mathrm{DATA}}+\mathrm{T}_{\mathrm{ACK}}\right)+\sum_{\mathrm{K}=1}^{\mathrm{m}-1} \gamma \mathrm{d}_{\max }^{\mathrm{r}} \\
& \times\left(\mathrm{T}_{\mathrm{RTS}}+\mathrm{T}_{\mathrm{CTS}}\right)+\sum_{\mathrm{k}=1}^{\mathrm{M}-1} \mathrm{P}_{\mathrm{r}} \times\left(\mathrm{T}_{\mathrm{DATA}}+\mathrm{T}_{\mathrm{ACK}}+\mathrm{T}_{\mathrm{RTS}}+\mathrm{T}_{\mathrm{CTS}}\right)
\end{aligned}
$$

where, $\mathrm{P}_{\mathrm{r}}$ is the receiving energy per second and is assumed that be the same for all types of packet.

Adhoc on demand multipath reliable and energy aware quality of service routing protocol: In Reliable and Energy Aware QoS routing protocol (REQR) (Thilagavathe and Duraiswamy, 2010) multiple paths are found based on the QoS constraints link reliability and energy consumption. It selects the path which satisfies the link reliability constraint (LR $\left.>\mathrm{LR}_{\mathrm{THRESHOLD}}\right)$. From the selected paths it chooses the path satisfying the constraint $\left(\mathrm{E}_{\mathrm{SD}}<\mathrm{E}_{\mathrm{THRESHOLD}}\right)$. But the link reliability metric is estimated by the assumption of MANET nodes which has exponential property (Jiang, 2004). The exponential nodes may not guarantee the link availability during the predicted period due to the following properties of exponential distribution: 
- It is memoryless, because each subsequent event is completely independent from the previous events

- It always modeling the constant failure rate

To overcome these problems, an analytical expression is formed in Eq. 1 to predict the link reliability using weibull distribution which improves the link reliability estimation. Normally, a weibull distribution is preferred to predict the reliability because the nodes have identical weibull distribution where the hazard rate is a function of the time.

An AOMP-REQR is a multipath routing protocol that can be designed mainly for highly dynamic energy deficient ad hoc networks where link failure and route break occurs frequently. When a single path on demand routing protocol is used in such networks, a route rediscovery is needed in response to every route break. Each route discovery is associated with high overhead and latency. This inefficiency can be avoided by using multipath routing protocol. Maintaining a routing table for multipath makes the protocol complex. The complexity can be reduced by selecting few of the best paths from the available multi paths based on multiple constraints and routing data packets through them. The AOMDV route discovery procedure is modified to enable the selection of the paths and for the computation of link reliability and total energy consumed. The RREQ and RREP message includes the following additional fields namely, Link reliability and Total Energy Consumed. The following expressions are used to calculate the QoS metrics:

$$
\begin{aligned}
& \mathrm{LR}_{\mathrm{SD}}=\sum_{\mathrm{i} \in \mathrm{p}(\mathrm{S}, \mathrm{D})} \mathrm{CR}(\mathrm{l}) \mathrm{E}_{\mathrm{SD}}=\sum_{\mathrm{k}=1}^{\mathrm{m}-1} \gamma \times \mathrm{d}_{\mathrm{k}(\mathrm{k}+1)} \times\left(\mathrm{T}_{\mathrm{DATA}}+\mathrm{T}_{\mathrm{ACK}}\right)+ \\
& \sum_{\mathrm{K}=1}^{\mathrm{m}-1} \gamma \mathrm{d}_{\text {max }}^{\mathrm{r}} \times\left(\mathrm{T}_{\mathrm{RTS}}+\mathrm{T}_{\mathrm{CTS}}\right)+\sum_{\mathrm{k}=1}^{\mathrm{M}-1} \mathrm{P}_{\mathrm{r}} \times\left(\mathrm{T}_{\mathrm{DATA}}+\mathrm{T}_{\mathrm{ACK}}+\mathrm{T}_{\mathrm{RTS}}+\mathrm{T}_{\mathrm{CTS}}\right)
\end{aligned}
$$

After determining the multiple link disjoint stable paths, the primary path (i.e., path to be used) will be selected using a following evaluation method called path selection parameter:

$$
\mathrm{P}_{\text {selection }}=\frac{\mathrm{LR}_{\mathrm{SD}}}{\mathrm{E}_{\mathrm{SD}} \times \mathrm{HC}_{\mathrm{SD}}}
$$

where, $\mathrm{HC}_{\mathrm{SD}}$ is hop count:

$$
\mathrm{HC}_{\mathrm{SD}}=\text { Number of nodes }
$$

For all the available paths in the source the path selection parameter value is determined and the path which has the highest path selection parameter value is used as primary path for transmitting data.
In this protocol, while the source node sending data along the route, routes may break due to node mobility or link and node failures. Then the route maintenance must be performed. Immediately, ROUTE ERROR (RERR) packet will be send to the source node for error processing. In the phase of error processing the source node removing the relevant entries in the route table that uses the broken links/nodes. Then the source node begins to transmit data through the next best routes available.

In addition while transmitting data through the primary path, if the link reliability of any one or more links may be less than the minimum threshold value (i.e., $\mathrm{LR}_{\mathrm{SD}}<\mathrm{LR}_{\text {THESHOLD }}$ ) then the ROUTE ERROR (RERR) packet is sent to the source node along the route. As mentioned above the source removes all the routes that include the failed links and reroute the transmission through the next best route. Normally, route error packet is sent when there is route break and the recovery is carried out after that. Here, the recovery is performed proactively before the route break or route failure.

\section{RESULTS}

Simulation: The simulation of the protocol has been performed using the NS2 Network simulator USC/ISI. The various parameters of simulation are shown in Table 1.

The performance of an Multipath Reliable and Energy Aware Quality of Service Routing for Mobile Adhoc Networks (AOMP-REQR) is compared with REQR (Thilagavathe and Duraiswamy, 2010) and AOMDV. The performance of the protocol is evaluated mainly based on the following metrics.

Control overhead: The control overhead is defined as the total number of routing control packets normalized by the total number of received data packets.

Average end-to-end delay: The end-to-end-delay is averaged over all surviving data packets from the sources to the destinations.

Table 1: Simulation parameters

\begin{tabular}{ll}
\hline Network size & $1000 \times 1000 \mathrm{~m}$ \\
\hline No. of nodes & 50 \\
Simulation time & $100 \mathrm{sec}$ \\
Packet size & $512 \mathrm{bytes}$ \\
Mobility model & Random way point \\
Node speed & $5 \mathrm{~m} \mathrm{sec}$ \\
Pause time & $0,10,20,30,40$ \\
Transmission range & $250 \mathrm{~m}$ \\
Node transmission & $2 \mathrm{Mbps}$ \\
\hline
\end{tabular}


Packet Delivery Ratio: It is the ratio of the number of packets received successfully and the total number of packets sent.

Drop: It is the number of packets dropped.

Average Energy: It is the average energy consumption of all nodes in sending, receiving and forward operations.

\section{DISCUSSION}

Figure 1 depicts that the Routing overhead for REQR, AOMDV and an AOMP- REQR and the routing overhead of AOMP- REQR is comparatively smaller than the other two protocols. The packet delivery ratio of an AOMP- REQR is much better than REQR and AOMDV in all scenarios as shown in Fig. 2 because the less path failure causes less traffic and less packet loss. Figure 3 shows that the delay of an AOMP- REQR decreases drastically. From Fig. 4, it is inferred that the energy consumption is slightly lesser than the REQR and AOMDV. In Fig. 5, the delay of an AOMP- REQR decreases considerably than REQR and AOMDV. Figure 6 compares the results of overhead and an AOMP- REQR results better performance than REQR and AOMDV. Figure 7 shows better packet delivery ratio of AOMP-REQR compared to REQR and AOMDV due to less path failure and less packet loss.

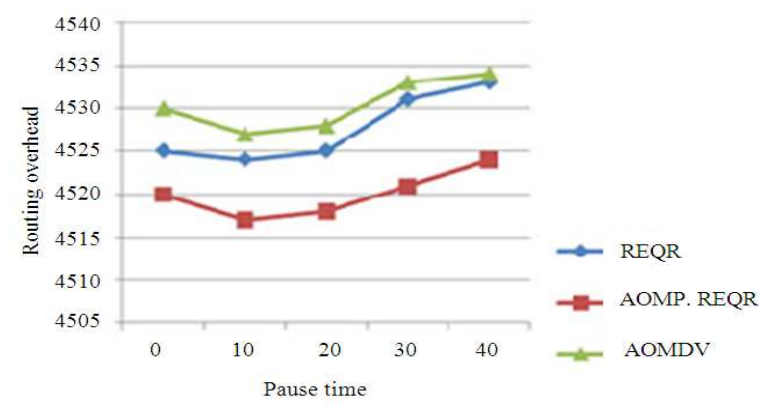

Fig. 1: Pause time Vs routing overhead

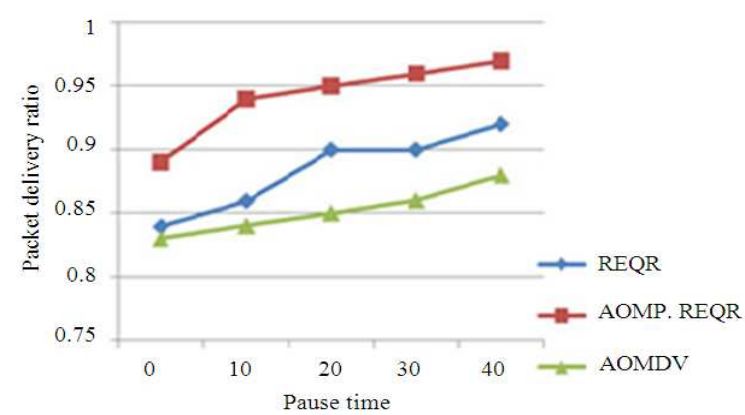

Fig. 2: Pause time Vs delivery ratio

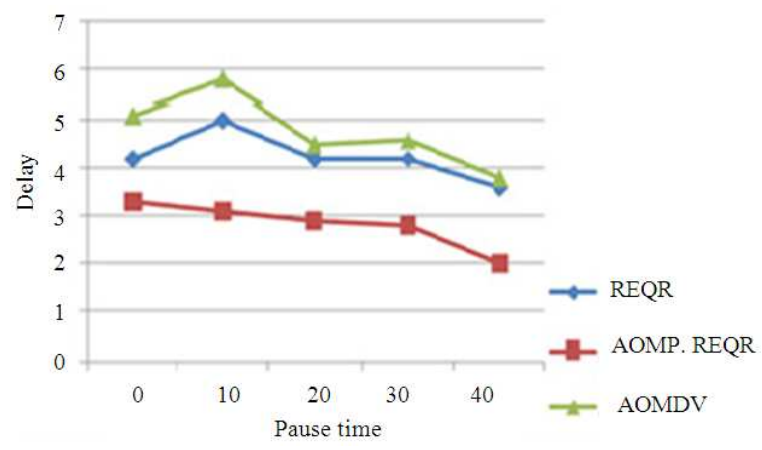

Fig. 3: Pause time Vs delay

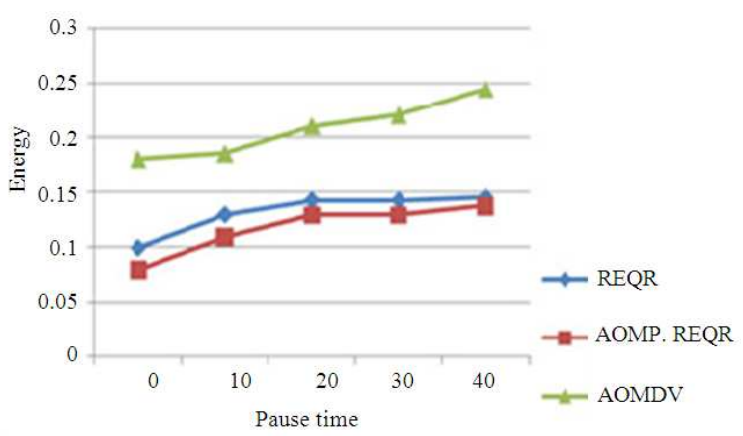

Fig. 4: Pause time Vs energy

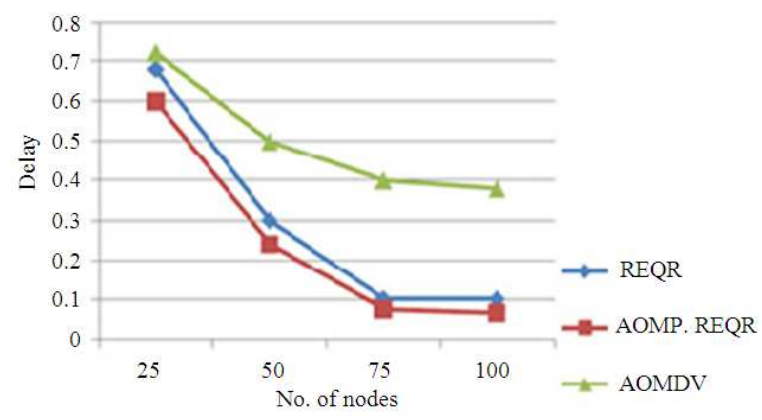

Fig. 5: No. of nodes Vs delay

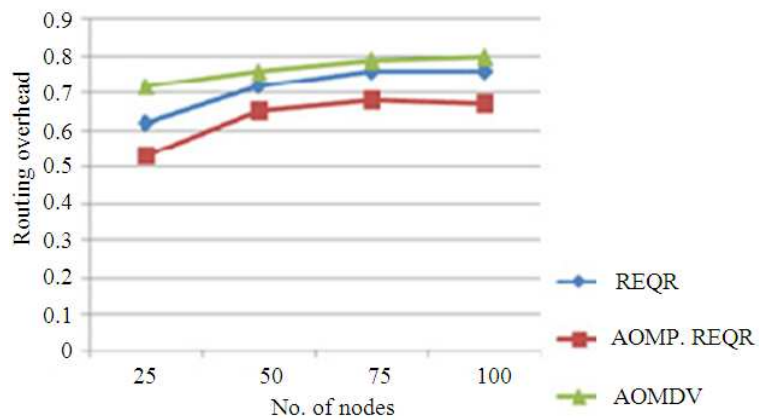

Fig. 6: No. of nodes Vs routing overhead 


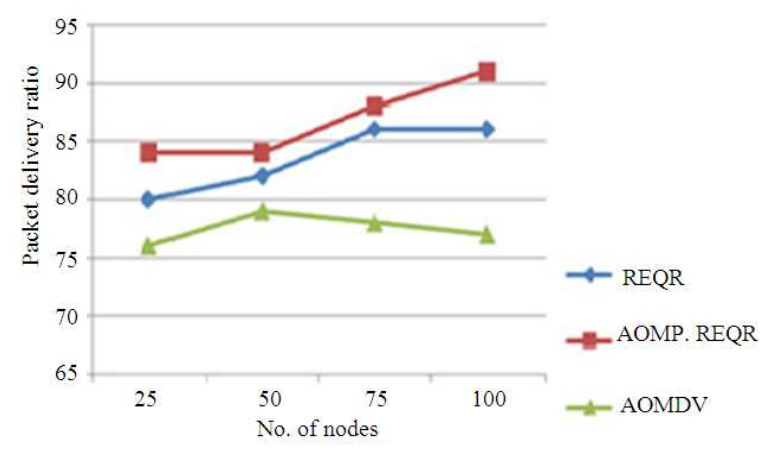

Fig. 7: No. of nodes Vs delivery ratio

\section{CONCLUSION}

Link failure due to node mobility or energy level always hinders communication and increases the number of packet loss which in turn affects the Quality of Service. We have proposed and investigated prediction based link reliability estimation with weibull nodes CR $\left(t_{p}\right)$ for MANET and an Adhoc On Demand Multipath Reliable and Energy Aware Quality of Service Routing for MANET (AOMP-REQR), which modifies the existing multipath routing protocol AOMDV to find the multiple stable link disjoint paths between the nodes using the QoS metrics Link Reliability and Energy Consumed. From the selected paths, based on the path selection metric the best path is selected and that is used for further transmission. During transmission of data through the primary path, if reliability of the link is less than the threshold value immediately route error packet is transmitted to source node. Hence the route is monitored using reliability prediction of the link, route break/route error is avoided. Simulation results show that the proposed protocol achieves better packet delivery ratio with reduced energy consumption and delay.

\section{REFERENCES}

Jiang, S., 2004. An enhanced prediction-based link availability estimation for MANETs. IEEE Trans. Commun., 52: 183-186. $\quad$ DOI: 10.1109/TCOMM.2003.822739

Jiang, S., D. He and J. Rao, 2001. A prediction-based link availability estimation for mobile ad hoc networks. Proceedings of the $20^{\text {th }}$ Annual Joint Conference of the IEEE Computer and Communication Societies, Apr. 22-26, IEEE Xplore Press, Anchorage, AK , USA., pp: 17451752. DOI: $10.1109 /$ INFCOM.2001.916672
Lim, G., K. Shin, S. Lee, H. Yoon and J.S. Ma, 2002. Link stability and route lifetime in ad-hoc wireless networks. proceedings of the International Conference on Parallel Processing Workshops, Aug. 21-21, IEEE Xplore Press, Seoul, pp: 116123. DOI: 10.1109/ICPPW.2002.1039720

Maleki, M., K. Dantu and M. Pedram, 2003. Lifetime prediction routing in mobile ad hoc networks. IEEE Wireless Commun., 2: 1185-1190. DOI: 10.1109/WCNC.2003.1200539

Marina, M.K. and S.R. Das, 2001. On-demand multipath distance vector routing in ad hoc networks. Proceedings of IEEE International Conference on Network Protocols, Nov. 11-14, IEEE Xplore Press, OH. USA., pp: 14-23. DOI: 10.1109/ICNP.2001.992756

Meghanathan, N., 2007. Stability-energy consumption tradeoff among mobile ad hoc network routing protocols. Proceedings of the 3rd International Conference on Wireless and Mobile Communications, Mar. 4-9, IEEE Xplore Press, Guadeloupe, pp: 9-9. DOI: 10.1109/ICWMC.2007.85

Perkins, C.E. and E. M. Royer, 1999. Ad-hoc ondemand distance vector routing. Proceedings of the 2nd IEEE Workshop on Mobile Computing Systems and Applications, Feb. 25-26, IEEE Xplore Press, New Orleans, LA, USA., pp: 910100. DOI: 10.1109/MCSA.1999.749281

Scott, K. and N. Bambos, 1996. Routing and channel assignment for low power transmission in PCS. Proceedings of the $5^{\text {th }}$ IEEE International Conference on Universal Personal Communication, Sep. 29-Oc. 2, IEEE Xplore Press, Cambridge, MA, USA., pp: 498-502. DOI: 10.1109/ICUPC.1996.562623

Singh, S., M. Woo and C.S. Raghavendra, 1998. Power-aware routing in mobile ad hoc networks. Proceedings of the 4th Annual ACM/IEEE International Conference on Mobile Computing and Networking, (MCN'98), ACM New York, NY, USA., pp: 181-190. DOI: 10.1145/288235.288286

$\mathrm{Su}, \mathrm{W}$. and M. Gerla, 1999. IPv6 flow handoff in ad hoc wireless networks using mobility prediction. Proceeding of the IEEE Globel Telecommunication Conference, Dec. 5-9, IEEE Xplore Press, Rio de Janeireo, Brazil, pp: 271-275. DOI: 10.1109/GLOCOM.1999.831647

Tan, L., P. Yang and S. Chan, 2007. An error-aware and energy efficient routing protocol in manets. Proceedings of 16th International Conference on Computer Communications and Networks, Aug. 13-16, IEEE Xplore Press, Honolulu, HI, pp: 724729. DOI: $10.1109 /$ ICCCN.2007.4317903 
Thilagavathe, V. and K. Duraiswamy, 2010. Reliable and energy aware qos routing protocol for mobile ad hoc networks. Int. J. Comput. Sci. Inform. Sec. 8: 272-278.

Toh, C.K., 2001. Maximum battery life routing to support ubiquitous mobile computing in wireless ad hoc networks. IEEE Commun. Magaz., 39: 138147. DOI: $10.1109 / 35.925682$

Balaji, V. and V. Duraisamy, 2011. Varying overhead ad hoc on demand vector routing in highly mobile ad hoc network. J. Comput. Sci., 7: 678-682. DOI: 10.3844/jcssp.2011.678.682
Maruthamuthu, S. and S. Sankaralingam, 2011. Energy aware multiple constraints quality of service routing protocol with dynamic mobility prediction for mobile ad hoc networks. J. Comput. Sci., 7: 892-901. DOI: $10.3844 /$ jcssp.2011.892.901 